\title{
Simulation Training in Urology: State of the Art and Future Directions
}

This article was published in the following Dove Press journal:

Advances in Medical Education and Practice

\section{Katie Lynn Canalichio ${ }^{1,2}$ \\ Claudia Berrondo (iD ${ }^{3}$ \\ Thomas S Lendvay ${ }^{1,2}$ \\ 'Pediatric Urology, Seattle Children's Hospital, Seattle, WA, USA; ${ }^{2}$ Urology, University of Washington, Seattle, WA, USA; ${ }^{3}$ Pediatric Urology, University of Nebraska Medical Center, Omaha, NE, USA}

\section{Video abstract}

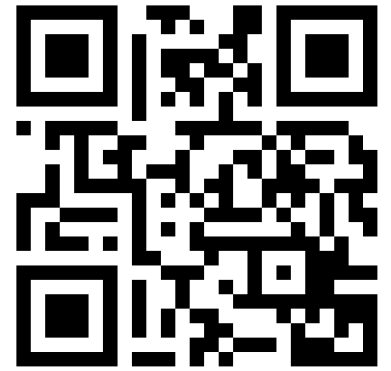

Point your SmartPhone at the code above. If you have a QR code reader the video abstract will appear. Or use: https://youtu.be/ZYYWWREEypw8
Correspondence: Katie Lynn Canalichio Pediatric Urology, Seattle Children's Hospital, OA.9.220 PO Box 537I, Seattle, WA 98।45-5005, USA

Tel + I 2069876913

Fax + I 2069873155

Email Katie.Canalichio@seattlechildrens. org

\begin{abstract}
There has been a major shift from the old paradigm of 'see one, do one, teach one' in medical training due in large part to resident work-hour restrictions and required oversight in the operating room. In response to this, advancements in technology have allowed for the introduction of more objective measures to assess the skill competency and proficiency of surgical trainees. Patient safety and trainee well-being are important drivers for this new model, and so surgical training programs are adopting simulation into their curriculum. Urology is uniquely positioned at the forefront of new emerging technologies in surgery, because of the field's commitment to safe and efficient minimally invasive surgery and endourological procedures. Due to these technically challenging procedures, urological training must incorporate these educational technologies to allow for objective skills assessment, skills transfer, and ultimately providing optimal patient care with the production of proficient and competent urological trainees.
\end{abstract}

Keywords: surgical education, virtual reality, 3D printing, evaluation, credentialing, crowdsourced

\section{Introduction}

More than 400,000 deaths per year in the United States are due to errors in medicine, and this accounts for the third leading cause of death. ${ }^{1,2}$ Mandated reporting for medical errors varies by state. And despite this, the US has higher reported medical errors than other developed countries. ${ }^{3}$ Surgical errors are common, but largely attributable to surgical error, and thus potentially preventable. ${ }^{4}$ Furthermore, surgical technique has been directly related to patient outcomes. ${ }^{4,5}$

Simulation allows for developing and practicing surgical skills without risk to patient safety. This 'learner-centered' approach is particularly important in the education of medical students, surgical residents and fellows. Over the past two decades, there have been many advancements in the technology available to objectively assess surgical skills. In addition, implementation of work-hours restrictions and the need for direct resident supervision in the operating room have created a new demand for pre-clinical technical and cognitive skills training through simulation. Urological training is currently assessed using a combination of direct preceptor oversight, case logs, written and oral boards. With the increasing availability of simulation models, more and more training programs are incorporating surgical simulation with objective benchmarks into their training curricula. This model allows for safe and efficient training curricula with the ability to evaluate skills in an objective and consistent manner. Minimally invasive surgery in 
particular lends itself to objective skill markers through video capture and movement tracking.

A variety of surgical simulation platforms have been developed to aid in surgical training including open surgery, endoscopic surgery, laparoscopic surgery and robotassisted laparoscopic surgery. Different platforms allow for a variety of surgical scenarios including task-based simulations, procedure-based simulation, reality-based simulation and virtual reality-based simulation. Simulation can also help trainees reach proficiency in surgical procedures using simulation-gained technical skills that can be applied to live-patient surgery.

Our aim in this article is to review current trends in surgical simulation in urology and discuss future directions. A literature search covering the past 10 years was conducted with keywords: "urology", "simulation", "education", and "surgical training". We included articles from this timeframe with special emphasis on the past 3 years. Preference was given to articles mentioning novel use of simulation tools in the field of urology. Additional preference was given to articles with higher levels of evidence. Exclusion criteria were articles that were not available in the English language.

\section{Simulation Platforms}

A variety of surgical simulators have been developed and utilized over the past two decades. Surgical simulation tools are based on surgical approach (open, endoscopic, laparoscopic, robotic), reality-based or virtual-reality based, and task-oriented or procedure-oriented platforms. There exist hybrids of these such as augmented reality or full immersion scenarios (Figure 1). ${ }^{6,7}$ Surgical simulation models have been validated in different settings for varying skill levels (from medical students to experienced surgeons) in multiple surgical specialties.

\section{Endoscopic Platforms}

Endoscopic training platforms can be utilized for both lower urinary tract and upper urinary tract urological procedures. Some common procedure-based platforms include simulation for cystoscopy, transurethral resection of the prostate (TURP), transurethral resection of bladder tumor (TURBT), ureteroscopy, and percutaneous nephrolithotomy (PCNL). ${ }^{6}$ The use of training platforms is particularly useful for PCNL, where the learning curve has been described in the literature as 45-60 cases for competency, but up to 115 cases need for proficiency. ${ }^{6}$

\section{Laparoscopic Platforms}

Physical box or video trainers have been the primary simulators available in laparoscopic surgery. These utilize real laparoscopic surgical instruments, allowing for the learner to become better acquainted with the tools (Figure 2). The learner can use the instruments to complete a variety of tasks using different surgical instruments which include passing objects, cutting, suturing and tying. These laparoscopic platforms have shown benefits in laparoscopic skills transfer in surgical trainees. Steigerwald et al demonstrated that surgical residents using reality-based or virtual realitybased laparoscopic trainers improved their scores during simulation and the live operating room setting. However, there was no significant difference in the performance of the residents using one system compared to the other. ${ }^{8}$

\section{Robotic Platforms}

Robotic simulation platforms have become increasingly popular in recent years. Currently available virtual simulators for robotic surgery are the Surgical Education Platform (SimSurgery, Oslo, Norway), Robotic Surgical System (Simulated Surgical Systems, San Jose, CA,

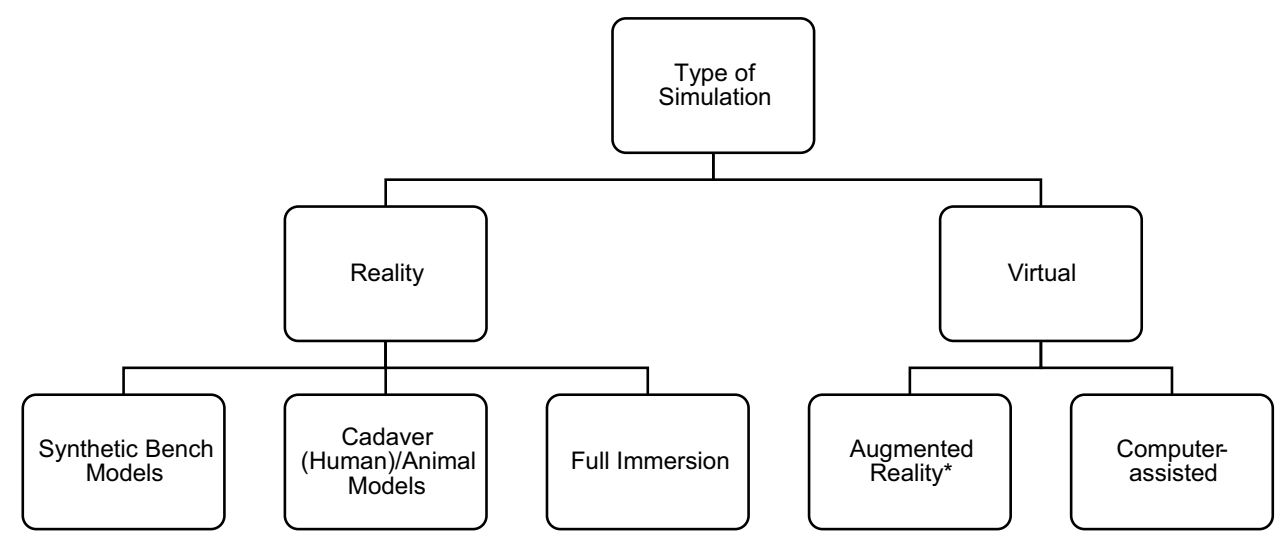

Figure I Type of simulation models in surgical training. *Hybrid experience. 

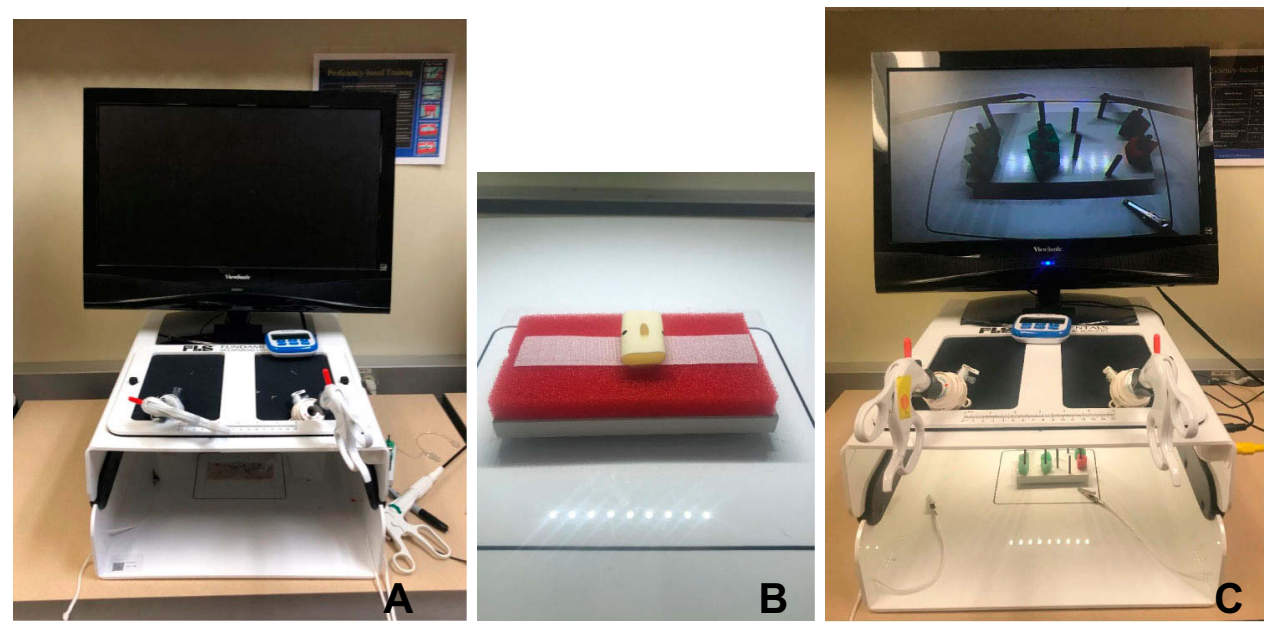

Figure 2 Examples of Task-Based Simulation. (A) Laparoscopic Box Trainer. (B) Suturing. (C) Peg Transfer.

USA), dV-Trainer (Mimic, Seattle, WA, USA), da Vinci Skills Simulator (Intuitive Surgical, Sunnyvale, CA, USA), ProMIS Simulator (Haptica, Ireland), and RobotiX Mentor (3D Systems, Simbionix Products, Cleveland, OH, USA). ${ }^{7,9}$ These simulators are not without cost; the most expensive costing about $\$ 160,000$ US dollars. The dV-Trainer was introduced in 2007, is the only unit with haptic feedback, and is stand-alone from the da Vinci console. The da Vinci Skills Simulator introduced in 2011 has a lower cost (\$90,000 US dollars), but is limited because it is not stand-alone, meaning it cannot be used without the surgical console. ${ }^{6}$ The advantage of these simulators is that procedure-specific modules exist to exercise the competence of a learner's ability by surgery case type.

\section{Task-Based Simulation}

Task-based simulation is the simplest, and most commonly utilized form of simulation in surgical training and can be used with both reality and virtual reality simulators. The tasks are not specific to any specific operation, but rather focus on developing fundamental skills. The tasks are completed with inanimate objects and focus on skills such as hand-eye and left-to-right-hand coordination, grasping, transferring, cutting, and suturing. Some common tasks performed include peg transfer, pattern cutting, needle driving, suturing and knot tying. Several publications have demonstrated that task-based simulation improves surgical skills in the operating room setting. Dawe et al demonstrated the transferability of surgical skill from surgical simulation training to the live patient setting in a systematic review. This review included 14 studies on laparoscopic simulation, 13 studies on endoscopic simulation and 7 studies on other types of procedures. Most of the studies reviewed showed improved performance for participants with simulation training compared to those without simulation training. ${ }^{10}$ In a study by Sethi et al, 20 participants (including experienced surgeons, trainees and medical students) perform basic skills (ie ring and cone, string walk, and letterboard) on a virtual reality simulator for the robot (dV-Trainer). Authors were able to conclude face and content validity, and all participants found this model realistic and easy to use. ${ }^{11}$

\section{Procedure-Based Simulation}

Procedure-based simulation gives trainees the opportunity to perform parts of or an entire surgical procedure in a simulated environment. Understandably, the difficulty with this type of simulation lies in the capacity to replicate the operative experience. ${ }^{12}$ The gold standard for this has been considered the cadaver model followed by the animal model, which is limited due to financial and ethical constraints. ${ }^{7}$ There exist many synthetic bench models in use for this purpose; these are typically designed to mimic a specific organ with a known pathological condition. Several publications that have shown validity and reliability using these models for specific steps of a procedure, such as with intracorporeal suturing. ${ }^{7}$ A recent example includes a laparoscopic ureteral reimplantation model developed by Millan et al in 2018. The goal for this model was to be anatomic, ergonomic, and low-cost (cost of materials was \$25). This model employed the Lich-Gregoir technique for pediatric ureteral reimplantation. Surgeon participants $(n=34)$ responded to a survey 
afterwards, and based on a perceived lack of training in $100 \%$ of respondents at their own institutions, they felt this would allow for practice of a technique that is not commonly employed, has a steeper learning curve, but with potential for improved outcomes in patients. ${ }^{13}$ Additional investigation of this model is needed to determine face and construct validity. Other examples include suprapubic catheter insertion models, ${ }^{14}$ abdominal phantom model for ultrasound-guided percutaneous renal access, ${ }^{15}$ and benign prostatic hyperplasia (BPH) prostate models for transurethral resection of the prostate (TURP). ${ }^{7}$ An example of the latter is the Bristol TURP trainer (Limbs and Things Ltd., Bristol), which includes a synthetic prostate and bladder mounted on a base, in which the trainee employs an actual resectoscope. ${ }^{16}$

Several examples of virtual reality procedure-based simulation exist in the literature as well. Moglia et al performed a systematic review of the literature on virtual reality simulators for robot-assisted surgery in 2016. Among these, Chowriappa et al randomized trainees into two groups: the intervention arm was instructed to perform procedure-based virtual reality training that included a specific step in a robotic surgical procedure versus a control group that was not given this exposure. The specific technology the authors used was a Hands-on Surgical Training (HoST) environment that uses augmented reality where an actual surgical procedure, in this case urethrovesical anastomosis, is transplanted onto a virtual simulator. Ultimately, this allows the trainee to take on the steps of an entire operation in this augmented reality, and also providing real-time feedback from within the framework. The intervention arm had overall higher scores and better performance compared to the control arm, and the majority found this platform similar to a real surgical procedure. ${ }^{12}$ Other urological procedures that are offered on this training platform are radical and simple prostatectomy, radical cystectomy, extended lymph node dissection, adrenalectomy, radical and partial nephrectomy. ${ }^{17}$ Additional virtual reality models exist for endourological cases, such as cystoscopy, transurethral resection of bladder tumors (TURBT) and TURP. Some of the virtual reality simulators available for TURP include the TURPsim (Simbionix/VirtaMed, Beit Goal, Israel) and the UroTrainer (Karl Storz, Tuttlingen, Germany). ${ }^{16,18}$

Whitehurst et al performed a randomized study to compare virtual reality simulation to a dry lab simulation in surgeons and trainees who had no previous exposure to robotic surgery. After randomization to one of these arms, the final performance was assessed with procedure completion in a live animal model. The authors found no difference in surgeon performance between the two groups and concluded that virtual reality simulation can be used for training in robotic surgery. ${ }^{19}$

\section{Emerging Technologies}

Three-dimensional (3D) printing has gained momentum since its introduction in the mid-1980s, and its role in urological training and surgery has been widely reported on in the past decade. This allows for constructing a 3D model in a layer-by-layer technique using a range of various materials. ${ }^{20}$ When creating an anatomical model, it is often acquired with CT or MRI diagnostic imaging, and a specialty trained individual, often a radiologist, is needed for final processing of the image before it is considered ready. ${ }^{20}$ Smith et al 2019 performed a review on the topic, identifying a large swath of urological procedures that some aspect of 3D printing has been used in, including percutaneous nephrolithotomy, partial nephrectomy, renal transplantation, laparoscopic pyeloplasty, prostate brachytherapy, TURBT, urethrovesical anastomosis, simulation, and "phantom organs". The available work on this has been largely low-level evidence, so there is a need for more investigation including randomized controlled trials. $^{20}$

Ghazi et al developed a full-immersion simulation for percutaneous nephrolithotomy using an anatomically correct $3 \mathrm{D}$ model. The authors were able to show face and content validity after testing this model in both urology and interventional radiology trainees as well as experienced practitioners. ${ }^{21}$ Cheung et al created a $3 \mathrm{D}$ model for laparoscopic pyeloplasty, and the authors were able to demonstrate validity as well between pediatric urology fellows and attending urologists. ${ }^{22}$ Van Renterghem et al described a 3D pelvic cadaver model with creation of a high fidelity and safe penile model that they consider not to be cost-prohibitive. This model was engineered with 3D printing, and designed for training in penile prosthetic surgery. ${ }^{23}$ These and other 3D models permit the use of high-validity, full-procedure simulation to be used in surgical training. There has also been an application in the real world with patient-specific models created to help surgeons prepare preoperatively for complex cases. ${ }^{9}$ Another emerging science with unique applications in surgery is augmented virtual reality systems that are employed at the patient level, and not just the training level. A recent article described the use of a MRI-based 
virtual reality surgical navigation tool that is overlaid in real time during robot-assisted radical prostatectomy. The authors were able to show feasibility by employing this adjunct tool during robot-assisted radical prostatectomy to help navigate surgical decision-making at key dissection points. At this time, the surgeon does have to step away from the console to visualize the contrived 3D models, but future directions would be for direct intraoperative use at the console level. ${ }^{24}$

\section{Role in Education and Certification}

In order to be considered as a tool for training and assessment, a surgical simulator must demonstrate validity, educational benefit, and cost-effectiveness. ${ }^{7}$ There are different measures of validity: face (experts and novices recognize the value of the tool), content (following expert evaluation), and construct (there is a measurable difference when using the tool between expert and novice). ${ }^{7,16}$ Fidelity is reflective of the level of realism the model has, but its necessity in simulation training is debated. ${ }^{16}$ Even higher standards need to be met for a tool to be considered for the assessment of surgeon skill. ${ }^{16}$ Ideally, the tool is able to show actual skill acquisition that is demonstrable in the clinical realm following simulation. ${ }^{9}$

The traditional model for evaluation of surgical skills is through direct observation; the Objective Structured Assessment of Technical Skill (OSATS) was introduced as an aid to objectively assess clinical competency. ${ }^{25}$ The Fundamentals of Laparoscopic Surgery (FLS) examination is currently a requirement to become board certified by the American Board of Surgery. Goh et al developed the Global Evaluative Assessment of Robotic Skills (GEARS) that assesses 6 domains (depth perception, bimanual dexterity, efficiency, autonomy, force sensitivity and robotic control) for credentialing in robot-assisted surgery. ${ }^{26}$ This tool has been validated at several institutions as part of an integrated curriculum for robotic surgery. $^{26}$ Beyond just one-on-one feedback, Lendvay et al have leveraged large groups of anonymous reviewers to provide a crowd-sourced assessment of surgical skills. ${ }^{27}$ Crowd-Sourced Assessment of Technical Skills (CSATS) represents the next level of objective assessment of surgical skills, correlates with expert reviews, and has shown cross-over into the prediction of patient outcomes. ${ }^{28}$

The inclusion of simulation within urology boot camps for new residents has shown improvements in knowledge, comfortability with instruments, and confidence of the trainee. ${ }^{6}$ Full immersion scenarios in training have also been described that mimic the entire operating room with integrated equipment, audio, and lighting. ${ }^{7}$ The concept of surgical warm-up prior to an operation has grown as well: Lendvay et al performed a randomized controlled trial that showed that expert surgeons benefited from a brief virtual reality warm-up session prior to robotic simulated tasks. ${ }^{29}$

At this time, there are no standard credentialing guidelines for specific procedures within the US. Hospitals look to the volume of cases the surgeon has done to determine credentialing eligibility. As simulation platforms continue to play a role in education, their role in credentialing will most likely increase as well.

\section{Conclusion}

Simulation provides a low-risk environment for repetition, development, and maintenance of surgical skills that is learner-centered. A range of simulation platforms have shown validity across the literature, and have also demonstrated improvement in surgical skills. There is ample evidence in the literature of the benefits, including faster procedure times and decreased errors, following simulation. With the changing needs of a surgical training program in the current environment of work-hours restrictions and focus on patient safety, simulation in training has been accepted as an adjunct for the surgical residency curriculum. ${ }^{30}$ We expect this trend to continue, and with it, an increasing role in surgical credentialing will mirror this. New technologies or applications of existing technologies are quickly changing the medical landscape, and have the potential to improve surgical training, credentialing, maintenance, and ultimately patient outcomes.

\section{Disclosure}

The authors report no conflicts of interest in this work.

\section{References}

1. James JT. A new, evidence-based estimate of patient harms associated with hospital care. J Patient Saf. 2013;9:122-128. doi:10.1097/ PTS.0b013e3182948a69

2. Heron M. Deaths: leading Causes for 2016. Natl Vital Stat Rep. 2018;67.

3. Anderson JG, Abrahamson K. Your health care may kill you: medical errors. Stud Health Technol Inform. 2017;234:13-17.

4. Jabbour N, Snyderman CH. The economics of surgical simulation. Otolaryngol Clin North Am. 2017;50:1029-1036. doi:10.1016/j. otc.2017.05.012

5. Birkmeyer JD, Finks JF, O'Reilly A, et al. surgical skill and complication rates after bariatric surgery. $N$ Engl J Med. 2013;369:1434-1442. doi:10.1056/NEJMsa1300625

6. Childs BS, Manganiello MD, Korets R. novel education and simulation tools in urologic training. Cur Urol Rep. 2019;20(12):81. doi:10.1007/s11934-019-0947-8 
7. Abboudi H, Khan MS, Guru KA, et al. Learning curves for urological procedures: A systematic review. BJU Int. 2014;114:617-629. doi:10.1111/bju.12315

8. Steigerwald SN, Park J, Hardy KM, Gillman LM, Vergis AS. Does laparoscopic simulation predict intraoperative performance? A comparison between the fundamentals of laparoscopic surgery and lapVR evaluation metrics. Am J Surg. 2015;209:34-39. doi:10.1016/j.amjsurg.2014.08.031

9. Song PH. Current status of simulation-based training and assessment in urological robot-assisted surgery. Investig Clin Urol. 2016;57:375-376. doi:10.4111/icu.2016.57.6.375

10. Dawe SR, Pena GN, Windsor JA, et al. Systematic review of skills transfer after surgical simulation-based training. $\mathrm{Br} J$ Surg. 2014;101:1063-1076. doi:10.1002/bjs.9482

11. Sethi AS, Peine WJ, Mohammadi Y, Sundaram CP. Validation of a novel virtual reality robotic simulator. J Endourol. 2009;23: 503-508. doi:10.1089/end.2008.0250

12. Chowriappa A, Raza SJ, Fazili A, et al. Augmented-reality-based skills training for robot-assisted urethrovesical anastomosis: A multi-institutional randomised controlled trial. BJU Int. 2015;11 5:336-345. doi:10.1111/bju.12704

13. Millán C, Rey M, Lopez M. LAParoscopic simulator for pediatric ureteral reimplantation (LAP-SPUR) following the Lich-Gregoire technique. J Pediatr Urol. 2018;14:137-143. doi:10.1016/j.jpurol.2017.11.020

14. Hossack T, Chris B-B, Beer J, Thompson G. A cost-effective, easily reproducible, suprapubic catheter insertion simulation training model. Urology. 2013;82:955-958. doi:10.1016/j.urology.2013.06.013

15. Filippou P, Odisho A, Ramaswamy K, et al. Using an abdominal phantom to teach urology residents ultrasound-guided percutaneous needle placement. Int Braz J Urol. 2016;42:717-726. doi:10.1590/ S1677-5538.IBJU.2015.0481

16. Brewin J, Ahmed K, Khan MS, Jaye P, Dasgupta P. Face, content, and construct validation of the Bristol TURP trainer. J Surg Educ. 2014;71:500-505. doi:10.1016/j.jsurg.2014.01.013

17. HOST. Simulated Surgicals, LLC n.d. Available from: http://www. simulatedsurgicals.com/host/. Accessed January 7, 2020.

18. Schulz GB, Grimm T, Kretschmer A, Stief CG, Jokisch F, Karl A. Benefits and limitations of transurethral resection of the prostate training with a novel virtual reality simulator. Simul Healthc. 2019. doi:10.1097/SIH.0000000000000396

19. Whitehurst SV, Lockrow EG, Lendvay TS, et al. Comparison of two simulation systems to support robotic-assisted surgical training: a pilot study (swine model). J Minim Invasive Gynecol. 2015;22:483-488. doi:10.1016/j.jmig.2014.12.160
20. Smith B, Dasgupta P. 3D printing technology and its role in urological training. World J Urol. 2019. doi:10.1007/s00345-01902995-1

21. Ghazi A, Campbell T, Melnyk R, et al. Validation of a full-immersion simulation platform for percutaneous nephrolithotomy using three-dimensional printing technology. $J$ Endourol. 2017;31:13 14-1320. doi:10.1089/end.2017.0366

22. Cheung CL, Looi T, Lendvay TS, Drake JM, Farhat WA. Use of 3-dimensional printing technology and silicone modeling in surgical simulation: development and face validation in pediatric laparoscopic pyeloplasty. J Surg Educ. 2014;71:762-767. doi:10.1016/j.jsurg.2014. 03.001

23. van Renterghem K, Ghazi A. 3D pelvic cadaver model: a novel approach to surgical training for penile implant surgery. Int J Impot Res. 2019. doi:10.1038/s41443-019-0211-2

24. Mehralivand S, Kolagunda A, Hammerich K, et al. A multiparametric magnetic resonance imaging-based virtual reality surgical navigation tool for robotic-assisted radical prostatectomy. Turk J Urol. 2019;45:357-365. doi:10.5152/tud.2019.19133

25. Martin JA, Regehr G, Reznick R, et al. Objective structured assessment of technical skill (OSATS) for surgical residents. Br J Surg. 1997;84:273-278. doi:10.1002/bjs.1800840237

26. Goh AC, Goldfarb DW, Sander JC, Miles BJ, Dunkin BJ. Global evaluative assessment of robotic skills: validation of a clinical assessment tool to measure robotic surgical skills. J Urol. 2012;187: 247-252. doi:10.1016/j.juro.2011.09.032

27. Lendvay TS, White L, Kowalewski T. Crowdsourcing to assess surgical skill. JAMA Surg. 2015;150:1086. doi:10.1001/jamasurg. 2015.2405

28. Holst D, Kowalewski TM, White LW, et al. Crowd-sourced assessment of technical skills: an adjunct to urology resident surgical simulation training. $J$ Endourol. 2015;29:604-609. doi:10.1089/ end.2014.0616

29. Lendvay TS, Brand TC, White L, et al. Virtual reality robotic surgery warm-up improves task performance in a dry laboratory environment: a prospective randomized controlled study. J Am Coll Surg. 2013;216:1181-1192. doi:10.1016/j.jamcollsurg.2013.02.012

30. Minneti M, Baker CJ, Sullivan ME. the development of a novel perfused cadaver model with dynamic vital sign regulation and real-world scenarios to teach surgical skills and error management. J Surg Educ. 2018;75:820-827. doi:10.1016/j. jsurg.2017.09.020
Advances in Medical Education and Practice

\section{Publish your work in this journal}

Advances in Medical Education and Practice is an international, peerreviewed, open access journal that aims to present and publish research on Medical Education covering medical, dental, nursing and allied health care professional education. The journal covers undergraduate education, postgraduate training and continuing medical education including emerging trends and innovative models linking education, research, and health care services. The manuscript management system is completely online and includes a very quick and fair peer-review system. Visit http://www.dovepress.com/testimonials.php to read real quotes from published authors. 